Богоявленская Д.Б.

\title{
Еще раз о «Креативном поле»
}

В статье обощается 35-летний опыт использования метода «Креативное поле». Автор выделяет две плоскости проблем, связанных с его применением другими исследователями: непонимание новой парадигмы раскрытия природы творчества и принципов его исследования и индентификации. Описывается двухслойная модель по решению задач, подтверждающая представление С.Л. Рубинштейна о мышлении как познании, а не просто решении задач.

Приводятся принципы, соблюдение которых необходимо для реализации метода. Подробно анализируется сходство, еще больще отличие точки зрения автора от взглядов других отечественных ученых на природу творчества и метод «Креативное поле».

Ключевые слова: творчество, интеллектуальная активность, решение задач, проблемная ситуация.

Данная статья в определенном смысле юбилейная. К моменту выхода этого номера журнала «Креативному полю» исполнится 35 лет. Возраст для метода весьма солидный. По мнению науковедов новая идея получает свое распространение через 15 лет. Простая арифметика указывает на наличие сложностей внедрения метода в широкую практику. Глазами автора видятся, в первую очередь, две плоскости проблем, возникающих в применении метода другими исследователями.

В первую очередь здесь приходится сталкиваться с непониманием в целом новой парадигмы раскрытия самой природы творчества и отсюда принципов его исследования и индентифрикации. Эти трудности связаны именно с новой «идеологией» творчества, а не с недостатками умственных способностей или профессионализма моих коллег. Наличие в числе «непонимающих» ведущих специалистов в области психологии и, в частности психологии творчества, тому подтверждение. И именно поэтому анализ их «ошибок» носит всеобщий характер.

Вместе с тем, проводимый ниже анализ ошибочных толкований важен не только в теоретическом плане, а приобрел в настоящее время особую актуальность в связи с тем, что на основе ситуаций, интерпрети- 
руемых как аналогичные методу «Креативного поля», строятся психодиагностические методики, получающие распространение в практике.

В сборнике «Психодиагностика и школа» Таллин, 1980г. я обобщала те сложности в понимании принципов метода «Креативное поле», которые обнаружились в первое десятилетие.

Совсем неожиданным было тогда обнаружение, что принципы «Креативного поля» идентифицировались с тестами креативности. Наша критика теории Дж. Гилфрорда с 1977 г. (Наука и жизнь, 1977, № 2) надеюсь, это смешение сделало более не актуальным².

Вместе с тем, узловые моменты, носящие нагрузку know-how продолжают вызывать вопросы. Остановимся на этом подробнее.

Напомним, что наш подход к изучению творчества шел по пути отказа не только от традиционных методов исследования, но и отказа от стоящей за ними модели эксперимента, что потребовало построения новой модели (Богоявленская, 1969). Она, в отличие от модели проблемной ситуации, в которой мысль движется как бы в одной плоскости (решение заданной задачи), должна быть объемной, чтобы проявилась другая плоскость (область, пространство) для прослеживания хода мысли за пределами решения исходной задачи. В этом качестве может выступать система однотипных задач, содержащая ряд общих закономерностей. Такая система задач обеспечивает построение двухслойной модели деятельности. Первый, поверхностный слой - заданная деятельность по решению конкретных задач, и второй, глубинный слой, замаскированный "внешним" слоем и неочевидный для испытуемого, - это деятельность по выявлению скрытых закономерностей, которые содержит вся система задач, но открытие которых не требуется для их решения. Требование решить задачу выступает в качестве стимула мыслительной деятельности до тех пор, пока испытуемый не находит и не отрабатывает надежный и оптимальный алгоритм решения. Дальнейший анализ материала, который не диктуется "утилитарной" потребностью выполнить требование (решить задачу), мы и называем образно вторым слоем. Поскольку переход в этот слой осуществляется после требуемого решения задачи, по инициативе самого субъекта, то в этом и только в этом смысле можно говорить об отсутствии внешнего стимула этой деятельности.

\footnotetext{
${ }^{2} \mathrm{~B}$ тестах же на креативность продуцирование любого числа ответов непосредственно стимулировано требованием инструкции, которая предусматривает любое количество ответов, на которые способен испытуемый.Отождествление отсутствия внешнего требования с неопределенностью требования в тестах на креативность, требования «отсутствия потолка» с открытостью многих тестов, длительности исследования с неограниченностью отдельного опыта во времени - неправомерно.
} 
Однако познавательный поиск может стимулироваться не только внешними требованиями, но и чувством неудовлетворенности результатами собственной работы. Оно проявляется в ситуации, когда испытуемый не владеет достаточно надежным алгоритмом выполнения заданной деятельности. Напомним, что наш подход требует создания условий для изучения деятельности, осуществляемой не как ответ на стимул. Реализация этого требования (принципа) возможна именно в силу того, что второй слой не задан эксплицитно в экспериментальной ситуации, а содержится в ней имплицитно. Через имплицитную представленность реальности легко развести представление о первом и втором слоях. В первом слое - заданной деятельности - эксплицитное наличие условий задачи и ее требований задают имплицитную представленность искомого. Второй же слой задан имплицитно лишь абстрактной возможностью всеобщей взаимосвязи реальности. Но ни один из его структурных компонентов не эксплицирован для субъекта деятельности. Он вызывается к жизни и реально обнаруживает себя лишь как результат проявленной активности человека, истинного механизма подлинно оригинального результата, снимающего мистический ореол с явлений, которые ранее представлялись как спонтанные, ничем не детерминируемые.

Использование метода «креативного поля» позволяет диагностировать стремление к собственно познавательной деятельности. Закономерности, имеющиеся внутри системы задания, относительно несложны; если специально дать задание обнаружить их, это будет сделано без значительных усилий. Однако эти закономерности «скрыты» лишь тем, что открытие их не требуется для успешного решения предъявляемых задач и перед испытуемым не ставится цель их обнаружения; будет ли он действовать во втором слое - поле скрытых закономерностей - или нет, зависит исключительно от него самого.

И чем богаче этот "слой" деятельности, чем шире система закономерностей, чем четче их иерархия, тем большей диагностической и прогностической силой обладает конкретная экспериментальная методика. Поскольку возможности испытуемого могут быть обнаружены лишь в ситуации преодоления и выхода за пределы требований исходной ситуации, то ограничение, "потолок" может быть, но он должен быть преодолен, снят. Структура экспериментального материала должна предусматривать систему таких ложных, видимых "потолков" и быть более широкой, неограниченной. "Отсутствие потолка" в экспериментальном материале, относится, конечно, не к отдельно взятому заданию, а к системе в целом, которая заключает в себе возможность неограниченного движения в ней. При этом такое движение по преодолению ложных ограниче- 
ний, движение как бы по ступенькам, может быть шкалировано, что позволит сопоставить результаты работы. Потенциальное присутствие второго слоя в любой деятельности еще раз подтверждает представление С.Л. Рубинштейна о мышлении как познании, а не просто решении задач. Однако его экспликация в экспериментальном исследовании возможна лишь при условии выполнения перечисленных принципов в единстве. Они образуют метод, который мы условно назвали "Креативное поле" (Богоявленская, 1969, 1970, 1983(.

Самые большие трудности возникают в понимании отсутствия внутреннего оценочного стимула деятельности. Познавательный поиск может стимулироваться не только внешними требованиями, но и чувством неудовлетворенности результатами собственной работы. Он проявляется в ситуации, когда испытуемый не владеет достаточно надежным алгоритмом выполнения заданной деятельности. Но такая мыслительная деятельность не может рассматриваться в качестве критерия интеллектуальной активности, понимаемой нами как продолжение мышления за пределами требований заданной ситуации. Интеллектуальная активность может однозначно рассматриваться как причина перехода во второй слой креативного поля только при условии наличия у испытуемого надежного оптимального алгоритма. Игнорирование этого требования иногда приводит к заблуждению, будто любая система однотипных задач сама по себе представляет первый слой «креативного поля». Действительно, этот метод может быть реализован на различном материале, но только при соблюдении реализации всех трех принципов.

Уже в первые годы мне пришлось столкнуться с, казалось бы, невероятным фрактом: непониманием принципов метода прекрасными профессионалами, людьми с тончайшим интеллектом. Каждый раз, когда, казалось бы, понятый принцип «прикладывался» на свой объект (свой - в смысле тщательно проработанный и обладающий своей логикой, структурой с ее компонентами и их связями), этот принцип начинал жить по логике этого объекта и в нем сохранялись лишь внешние очертания, а содержание терялось. Самым парадоксальным был пример с В.Н. Пушкиным, в лаборатории которого я работала с момента поступления в аспирантуру Института. Идея «Креативного поля» схватывалась, но затем ее суть мистически исчезала, что удивляло даже моих аспирантов.

Психологический смысл проводимого Пушкиным эксперимента он лично описывает так: «Испытуемому предъявляется совокупность задач игры «5», которые по внешнему своему виду кажутся различными. На самом деле все они представляют собой модификацию одной и той же 
задачи. Испытуемый не предупреждается об этом единстве. В ходе эксперимента выясняется, обнаруживает ли испытуемый общность задач и если обнаруживает, то на какой задаче происходит такой процесс обобщения.

Таким образом, в этой последовательности задач содержится, по крайней мере, две возможности. Одна из них связана с решением каждой предъявляемой задачи. Эта возможность фрормулируется экспериментатором. Вторая же возможность - возможность найти общее во всех предъявляемых задачах - представляет собой возможность, лишь потенциально содержащуюся в данной совокупности задач, но латентную, скрытую от решающего задачу человека. Если испытуемый распознает общность всех задач, то он совершит дополнительную деятельность, которая не требовалась от него инструкцией эксперимента. В этом случае процесс обобщения может рассматриваться как показатель интеллектуальной активности человека.

Нетрудно увидеть и существенное различие в функции цели в этих двух видах деятельности. У представителя той группы, которая все экспериментальные задачи решала как отдельные, имеет место лишь ситуация поставленной ранее цели. У представителей группы, обнаруживающей общность задач, помимо поставленной извне цели, имеется своя гностическая, познавательная цель, определяющая их деятельность. Здесь, следовательно, имеет место то явление, которое мы назвали обобщающим целеполаганием.

Методика эксперимента состояла в следующем. Младшим школьникам предлагалось решать 16 задач игры «5». В качестве первой задачи ребенку давалась простая четырехходовая задача: преобразовать ситуацию передвигая фришки на пустую клетку (левая нижняя клетка исходной и конечной ситуации): 243 в 123

\section{5.}

На примере этой тренировочной задачи испытуемый знакомился с правилами игры «5»... После решения тренировочной серии, он решал основную совокупность эквивалентных задач.

При, казалось бы, внешнем сходстве наших экспериментальных ситуаций, здесь не учитывается именно тот момент, что передвижение фришек на свободное поле, не является психологически способом решения задачи. По сути - это лишь одно из условий данной модели (игры 5). Действительно, «Психологической основой решения этих экспериментальных задач является установление отношений между элементами исходной ситуации (фишками) и элементами конечной ситуации. При всем внешнем несходстве этих десяти экспериментальных задач соотношение 
фришек в каждой задаче одно и то же. Здесь испытуемый как бы решает одну и ту же задачу, которая может быть выражена в буквенной записи...обобщенной формулой. Весь процесс обобщения в данном эксперименте состоял в том, чтобы перейти от многих ситуаций с фришками, обозначенными с помощью цифр, к этой единой обобщенной формуле» [6, c. 80-81].

Однако это и есть способ, принцип решения задачи. Поэтому стоит уточнить те две реальные возможности, которые имеет испытуемый в данной экспериментальной ситуации, с нашей точки зрения.

Одна заключается в решении задач методом проб и ошибок. При фрормальном отношении к участию в эксперименте (и в зависимости от уровня умственных способностей) можно этим ограничиться. Но это требует большего времени и поэтому гораздо выгоднее, в конце концов, найти способ решения, т.е. «найти общее во всех предъявляемых задачах». Правда, способ можно найти и на первой задаче при определенных критериях качества, присущих испытуемому ${ }^{3}$. Навряд ли, нахождение способа решения необходимого для осуществления успешности и надежности деятельности можно считать «дополнительной деятельностью». Ну, а если способы решать новые задачи будут лежать на поверхности и не будут «латентными, скрытыми от решающего», то мы не сможет исследовать продуктивное мышление, для чего и разрабатывалась Пушкиным описанная методика.

Таким образом, нельзя утверждать, что описанный «процесс обобщения может рассматриваться как показатель интеллектуальной активности». Интерпретация Пушкиным, по нашему мнению, объясняется невольным игнорированием роли оценочного стимула. Было бы неверно думать, что любое обобщение при решении однотипных задач - это уже переход во второй слой креативного поля; на самом деле переход во второй слой осуществляется лишь в том случае, если он не побуждается внешними или внутренними оценочными стимулами. В противном случае мыслительный процесс остается в рамках первого слоя - заданной деятельности.

Значительно более тонкий анализ проведен В.А. Петровским, казалось бы, аналогичной ситуации. Методика Петровского, пожалуй, более других внешне схожа с «креативным полем». Поэтому наше определение феномена творчества как «интеллектуальная активность» и термин Петровского «надситуативная активность» воспринимаются

\footnotetext{
${ }^{3}$ При проведении аналогичных экспериментов только на проволочных головоломках Леонтьев А.Н. отмечал, что двоешники уходили сразу, если им удавалось случайно расцепить детали. Однако, отличники, получив результат, не уходили, не поняв способ решения.
} 
почти всеми как синонимы (см.: Дружинин // Психол. жур. 2000). Термин «надситуативная активность» введенный позже на 7 лет снял определенные недостатки термина «интеллектуальная активность». Он значительно более удачен, поскольку свободен от какой-либо модальности. Вместе с тем, сами феномены и ситуации, их проявляющие не идентичны. В методике «бескорыстного риска» нет второго слоя. Выбор есть, но он может стимулироваться тем, что в условиях задачи эксплицирована зона риска. Таким образом, тот же внутренний оценочный стимул, в зависимости от уровня притязаний испытуемого и пр. (что очень тонко описано В. Петровским), не позволяет ему действовать формально, а требует испытания своих возможностей.

Фактически по поводу этого же принципа (отсутствие внутреннего оценочного) возникают возражения и у В.Д. Шадрикова:

Соглашаясь с предложенным подходом в целом и с определением интеллектуальной инициативы, мы все же с большой настороженностью относимся к утверждению, что эта инициатива не связана с практическими нуждами и оценкой работы. При этом непонятно утверждение автора, что ИИ не связана с отрицательной оценкой, но выходит, что она связана с положительной оценкой. Чем обусловлено это различие положительной и отрицательной оценки? И далее, если в структуру интеллектуальной активности входят регуляторные процессы, то они не могут обходиться без оценки, положительной или отрицательной. Оценка лежит в основе регуляторных механизмов [7, с. 63].

В данном случае мы сталкиваемся с тем же феноменом. В.Д. Шадриков наиболее продуктивно в настоящее время разрабатывает широкий спектр теоретической проблематики, в том числе, теорию деятельности. С этой позиции «настороженность» по отношению к перечисленным нашим положениям, это весьма деликатная фрормулировка. Вместе с тем, те закономерности, которые характерны для протекания деятельности «снимаются», когда речь идет о ситуативно нестимулированной продуктивной деятельности. Так, мы знаем, что разрабатываются целые теории о механизмах стимуляции деятельности для повышения ее эффрективности. Но само определение феномена творчества исключает любой вид стимуляции, так как если есть стимуляция, то нет феномена нестимулированной деятельности. Практические нужды стимулируют деятельность по их удовлетворению, но творческий уровень ее выполнения определяется уже не направленностью человека на разрешение практической проблемы, а поглощенностью самой деятельностью по ее разрешению. Повторю: практическая проблема стимулирует деятельность, а мера вовлечения в саму дея- 
тельность, уже без рефлексии на результат, определяет возможность развития самой деятельности, т.е. творчество.

Шадрикову непонятно, почему ИИ не связана с отрицательной оценкой. Дело в том, что если испытуемый отрицательно оценивает свою деятельность (работает слишком долго, делает ошибки, не уверен в верности способа решения или вообще работает методом проб и ошибок), то это стимулирует его к поиску, анализу всей экспериментальной ситуации, для того, чтобы успешно осуществлять, предлагаемую ему деятельность. Естественно, что этот поиск качественно отличается от того процесса, когда испытуемый, имея надежный способ работы, в совершенстве овладев деятельностью (положительно оценивая свою работу), продолжает анализ всей экспериментальной ситуации уже по своей инициативе. Его детерминантой является лишь познавательная потребность.

И, наконец, вопрос об обязательной связи регуляторных процессов с их оценкой. Само утверждение совершенно справедливо. Наряду с умственными способностями регуляторные процессы обеспечивают успешность овладения деятельностью. Уровень их развития важен, так как без овладения деятельностью невозможно ее развитие. И, вместе с тем, регуляторные процессы это лишь один из компонентов системы, обладающей неаддитивным свойством неприсущим ее компонентам. Если ИИ мы определяем как уровень действия, который не носит характера ответа, то принцип подкрепления здесь не действует.

Говоря, что ИИ как способность к саморазвитию деятельности не объяснима лишь свойствами интеллекта, а рассматривается нами как свойство целостной личности, отражающее взаимодействие когнитивной и афффективной сфер в их единстве, я естественно не имела намерения возвращаться в парадигму Аристотеля, в чем меня фрормально справедливо упрекнул В.Н. Дружинин. Но есть текст, и есть контекст. Говоря о ИИ как свойстве личности, я пыталась изменить вектор рассмотрения проблемы: подчеркнуть, что творческие способности являются свойством более широкой системы, чем интеллект. На языке А.Н. Леонтьева их «функциональным органом» является вся личность. И поскольку в этом случае они (как таковые, а не их компоненты, смотри выше) не имеют своей мозговой локализации, я для заострения своей точки зрения утверждаю, что творческих способностей в традиционном смысле слова просто нет. В принципе, повторяя нашу классификацию, Дружинин включил в данный подход («Как таковых творческих способностей нет») А. Танненбаума, А. Олоха и А. Маслоу, что в принципе искажает идею. Правда, затем Владимир Николаевич меня 
все же от Танненбаума (у которого случай и везение так же определяет возможность творчества) отличает, признавая позицию Богоявленской стоящей особняком [5, с.102].

В силу заслуженной популярности, большого тиража его книг, мне приходится, как правило, встречаться с изложением моей позиции по текстам Владимира Николаевича. Поэтому хочу уточнить ряд формулировок. Определение моей позиции Дружинин формулирует следующим образом: «Творчество, с точки зрения Д.Б. Богоявленской, является ситуативно-нестимулированной активностью, проявляющейся в стремлении выйти за рамки заданной проблемы» [там же]. В этом предложении сразу три неточности.

Во-первых, я говорю о ситуативно-нестимулированной продуктивной деятельности, что для меня и есть «активность» - как «инициатива начала изнутри» по Бернштейну. Естественно, что при определении А.Н. Леонтьевым деятельности как целенаправленной активности замена терминов «Продуктивная деятельность» на «Активность» кажется правомерной, если не учитывать, что автор уделяет целый раздел в своей монографии анализу категории «Активность» (см. [2, 4]).

Во-вторых, стремление выйти за рамки заданной проблемы противоречит моему пониманию творчества и реализации его в методе «Креативное поле». Если говорить о стремлении, то это, напротив, стремление к решению заданной проблемы. Стремления выйти за ее рамки (в нашем случае это выход во второй слой) просто не может быть, так как человек не знает о его существовании, пока сам его не открыл. Как правило, на решении проблемы мышление обрывается, так как меняет свой объект. «Я прошел в сантиметре от великого открытия», - признался Адамар, решивший свою проблему. Собственно вся теория и посвящена раскрытию этого феномена - выходу за пределы...., а метод позволяет его непосредственно наблюдать [1, 2].

B-третьих, в предыдущем предложении я поставила многоточие, так как исследуемый мною феномен творчества заключается не в выходе за пределы заданной проблемы, как излагает это Дружинин, а в выходе за пределы требований исходной проблемной ситуации. Неискушенному читателю обе формулировки могут показаться в основном схожими: выходе за пределы что проблемы, что проблемной ситуации - одно и то же. Вместе с тем, принципиально новым в методе является выход именно за требования заданной ситуации. Дело в том, что решение любой проблемной ситуации можно рассматривать как выход за ее пределы, так как нахождение искомого, нового условия меняет ее. Для нас выход за пределы требований является принципиальным, так 
как пока не выполнены условия (задача не решена) вся мыслительная деятельность человека стимулирована. Решения исходной проблемной ситуации (выполнение ее требований) и является тем водоразделом, который для меня отличает продуктивное мышление от творческого процесса.

Вторая плоскость проблем связана с самой процедурой.

В своей традиционной форме метод «Креативное поле» занимает прочные позиции среди исследовательских и психодиагностических методик. За 34 года его валидность получила достаточно солидное экспериментальное подтверждение. Через эксперимент проведено около 8 тысяч испытуемых: около 6 тыс. учащихся 38 школ разных регионов страны с 1 по 11 класс и дошкольников, а также свыше 2 тыс. взрослых широкого спектра профессий.

Вместе с тем, структура методик данного метода, обеспечивающая высокую валидность и прогностичность метода, имеет свою оборотную сторону в сложности и трудоемкости самой процедуры.

Метод «Креативное поле» кроме того, что он выявляет способность субъекта к развитию деятельности за пределами исходных требований позволяет на его первом этапе оценивать умственные способности испытуемого как по параметрам обучаемости: обобщенности способа действия, его характера, переноса, экономичности и самостоятельности, так и по степени сформированности операционального и регуляторного аппарата: полноте анализа условий задачи, частичному анализу условий задачи; планированию (стратегии поиска): хаотическому, направленному, оптимальному. Поэтому полная процедура принципиально индивидуального эксперимента представляет собой минимум 5 серий, занимающих в среднем от 20 до 40 минут. В частности, это отвечает одному из принципов метода: длительности и многократности эксперимента, так как только многократность тестирования может контаминировать влияния привходящих фракторов и, главное, обеспечивает возможность овладения предлагаемой в эксперименте деятельностью. Лишь при условии максимальной отработки испытуемым надежного алгоритма можно судить о наличии или отсутствии способности к нестимулированному извне развитию деятельности, что отражает наше концептуальное раскрытие понятия «творческие способности». Таким образом, при всей обоснованности трудоемкости диагностической процедуры она препятствует внедрению метода в широкую практику. Это же делает ее не конкурентно способной по сравнению с тестами с сомнительной валидностью, но легко реализуемыми даже непрофрессионалами.

Но... Impavide progrediamur (будем идти вперед без колебаний). 


\section{Лumepamypa}

1. Богоявленская Д.Б. Интеллектуальная активность как проблема творчества. Ростов н/Д: Изд-во РГУ, 1983.

2. Богоявленская Д.Б. Метод исследования уровней интеллектуальной активности // Вопросы психологии. 1971. № 1.

3. Богоявленская Д.Б. Предмет и метод исследования творческих способностей // Психологический журнал. 1995. № 5.

4. Богоявленская Д.Б. Психология творческих способностей. М.: Академия, 2002.

5. Дружинин В.Н. Психология общих способностей. М., 1995.

6. Пушкин В.Н. Психология целеполагания и проблемы интеллектуальной активности // Вопросы психологии. 1977. № 5.

7. Шадриков В.Д. Введение в психологию: интеллект и творчество. М., 2004. 